\title{
La comprensión de las demostraciones matemáticas. Un estudio de revisión
}

\section{The comprehension of mathematical proof. A review study}

\author{
${ }^{a}$ David Andree Parada-Carrillo, ${ }^{b}$ Laura Daniela Pumarejo García, ${ }^{\mathrm{c} C e s a r}$ Augusto Hernández-Suárez, ${ }^{\mathrm{d}}$ Raúl \\ Prada-Núñez
aEstudiante Licenciatura en matemáticas, davidandreepc@ufps.edu.co, https://orcid.org/0000-0001-7572-2807, Universidad Francisco de Paula Santander, Cúcuta-Colombia
bEstudiante Licenciatura en matemáticas, https://orcid.org/0000-0002-8828-9317, Universidad Francisco de Paula Santander, Cúcuta- Colombia
'Magister en enseñanza de las ciencias, cesaraugusto@ufps.edu.co, https://orcid.org/0000-0001-9409-8341, Universidad Francisco de Paula Santander, Cúcuta-Colombia
`Magister en Educación Matemática, raulprada@ufps.edu.co http://orcid.org/0000-0001-6145-1786, Universidad Francisco de Paula Santander, Cúcuta-Colombia

Forma de citar: Hernández-Suárez, C. A. ., Prada-Núñez, R. ., Parada-Carrillo, D. A. ., \& PumarejoGarcía, L. D. . (2020). La comprensión de las demostraciones matemáticas. Un estudio de revisión. Eco Matemático, 11 (2), 100-110

Recibido: 10 Abril de 2020

Aceptado: 22 Mayo de 2020

\section{Palabras clave \\ Demostración; \\ Comprensión; \\ Comprensión de las \\ demostraciones; \\ Lectura de las \\ demostraciones; \\ Educación matemática \\ de pregrado}

\begin{abstract}
Resumen: En este artículo se realizó una revisión de la literatura relacionada con la comprensión de las demostraciones matemáticas en el ámbito de la educación superior. Para esto, se parte del concepto de comprensión, y posteriormente se vincula al de las demostraciones matemáticas mediante exploración de investigaciones que la hayan estudiado. Estos artículos han sido agrupados en cuatro categorías: investigaciones centradas en el cambio de presentación del contenido, investigaciones centradas en el cambio del modelo de evaluación, modelos cognitivos que trabajan la comprensión de las demostraciones y estudios que han aplicado el modelo de evaluación en educación superior. En este análisis se manifiesta la necesidad de crear líneas de investigación que se enfoquen en la comprensión de manera similar a las demás actividades demostrativas.
\end{abstract}

*Autor para correspondencia: cesaraugusto@ufps.edu.co

Doi: https://doi.org/10.22463/17948231.3201 


\author{
Keywords \\ Demonstration; \\ Comprehension; \\ Comprehension of \\ demonstrations; \\ Reading demonstrations; \\ Undergraduate \\ mathematics education
}

\begin{abstract}
In this article a review of the literature related to the comprehension of mathematical proofs in the field of higher education was carried out. For this purpose, the concept of comprehension is taken as a starting point, and then it is linked to that of mathematical proofs through the exploration of researches that have studied it. These articles have been grouped into four categories: research focused on the change in the presentation of content, research focused on the change in the evaluation model, cognitive models that work on the understanding of demonstrations, and studies that have applied the evaluation model in higher education. This analysis shows the need to create lines of research that focus on comprehension in a similar way to other demonstrative activities.
\end{abstract}

\section{Introducción}

La definición de comprensión desde la Real Academia de la Lengua Española (RAE, s.f, definición 2) es expuesta cómo: "facultad, capacidad o perspicacia para entender y penetrar las cosas", sin embargo, esta definición se encuentra muy limitada para entender su significado en el ámbito educativo. En la Encyclopedia of the Sciences of Learning se encuentra el concepto de comprensión del discurso, el cual se refiere a hacer inferencias para conectar ideas dentro como a través de contextos discursivos locales y globales (Sparks, 2012), estableciendo conexiones entre los anteriores con los nuevos, y así construir representaciones coherentes con los conceptos que se describen en un texto, para que la representación de estos pueda recuperarse, actualizarse, manipularse y aplicarse para responder a preguntas y resolver problemas.

En el caso de las matemáticas, cuando los estudiantes establecen relaciones con objetos matemáticos y producen narrativas respaldadas sobre el objeto en cuestión, generalmente se realizan de acuerdo con meta-reglas bien definidas, de esta forma están aprendiendo a manejar un discurso matemático (Ben-Zvi y Sfard, 2007). Al respecto, las concepciones de dichos objetos han tenido transformaciones, mostrando diferencias en el discurso y en el contexto en el que se desarrollan (Harel y Sowder, 2007); en relación con las demostraciones en matemáticas Balacheff (1987) hace la diferenciación entre explicación, prueba y demostración, donde la explicación es un discurso que pretende hacer inteligible el carácter de verdad, una prueba es una explicación aceptada por una comunidad determinada en un momento determinado y la demostración es una prueba con enunciados organizados mediante reglas de deducción.

Selden y Selden (2017) realizaron una revisión de la literatura existente acerca de la comprensión de las demostraciones matemáticas, dando como resultados una diferenciación entre cuatro conceptos que abarcan distintos aspectos relacionados a la actividad demostrativa, tales como la comprensión, construcción, validación y la evaluación de las demostraciones.

La comprensión de las demostraciones ha sido descrita en términos pragmáticos en el modelo de evaluación de Mejía-Ramos, Fuller, Weber, Rhoads y Samkoff (2012) yendo desde la comprensión local hasta una comprensión holística de una demostración matemática. Sin embargo, aún no existe un consenso sobre qué y cómo los estudiantes la comprenden, puesto que, por lo general la comprensión de los estudiantes se mide pidiéndoles que reproduzcan o 
hagan ligeros cambios al momento de demostrar un teorema similar, dando cómo resultados una visión superficial de la comprensión de los estudiantes (Conradie y Frith, 2000; Rowland, 2001; Schoenfeld, 1988 y Weber, 2011).

Otro término asociado en la actividad demostrativa es la construcción delas demostraciones y significa intentar construir pruebas correctas al nivel esperado de los estudiantes universitarios de matemáticas (Selden y Selden, 2016), o en palabras de Hodds, Alcock y Inglis (2014) generalmente se entiende que las tareas de construcción de las demostraciones simplemente se relacionan con la creación de un argumento que intenta probar un teorema dado. Como expresa Selden y Selden (2016) la validación de las demostraciones se ha descrito como la lectura y la reflexión sobre los intentos de prueba para determinar su exactitud.

Como último término asociado a la actividad demostrativa es la evaluación de las demostraciones, estas han sido descrita por Pfeiffer (2011) como la determinación de si una demostración es correcta y también qué tan buena es con respecto a una gama más amplia de características tales como claridad, contexto, suficiencia sin exceso, perspicacia, convencimiento o mejora de la comprensión.

Aunque existe este trabajo de revisión enfocado en el análisis de estos cuatro conceptos, aún no se ha realizado un trabajo que describa la forma en cómo la investigación ha trabajado específicamente en la comprensión de las demostraciones. Por ello se plantea como objetivo de este artículo realizar una revisión descriptiva de la literatura que permita analizar cuáles han sido las formas de investigar la comprensión de las demostraciones matemáticas en la educación superior desde la perspectiva de la enseñanza y el aprendizaje de los estudiantes.

\section{Metodología}

Teniendo en cuenta la clasificación dada por Guirao-Gori, Olmedo Salas, Ferrer Ferrandis (2008), el presente trabajo es una revisión descriptiva puesto que: "proporciona al lector una puesta al día sobre conceptos útiles en áreas en constante evolución" ( $p$. 6 ), adicionalmente es de tipo documental dado que el procedimiento implica el rastreo, organización, sistematización y análisis de un conjunto de documentos electrónicos (Sánchez Upegui, 2011) sobre el tema de comprensión de las demostraciones en estudiantes de educación superior.

Para la búsqueda de los documentos bibliográficos sobre el tema, se utilizaron las bases de datos: Google Scholar, Funes, Springer, Eric, Taylor y Francis Online, Semantic, Jstor, Science Direct, Researchgate, entre otros.

Los principales términos de búsqueda, se incluyeron las siguientes palabras claves: comprensión de las demostraciones, comprensión de la prueba, las demostraciones, the proof, comprehension proof, comprehension math proof $y$ comprehension proof pre-service teacher. Se utilizaron en diferentes combinaciones los anteriores términos, de modo que permitirá ampliar los criterios de búsqueda y encontrar más documentos.

En el momento de realizar la búsqueda de los documentos en las bases de datos anteriormente nombradas, se realizó la selección de documentos, posteriormente se adelantó una revisión a los artículos citados dentro de estos documentos, estableciéndose relaciones bidireccionales entre los aportes dados por los diferentes autores. Para la revisión, no se tuvo en cuenta los artículos que no estaban relacionados con el tema. Con el fin de organizar los documentos, se utilizaron las siguientes categorías de análisis tales como: título del artículo, autor, año, revista, problema y objetivo de investigación, teorías utilizadas y su aplicación, participantes (nivel educativo), diseño de la investigación, técnicas de recolección de información, técnicas de análisis de datos y los hallazgos encontrados. Una vez ordenada la información, se agruparon los artículos en cuatro enfoques. 


\section{Desarrollo - Estado del arte}

Entre los intentos de mejorar la comprensión de las demostraciones de los estudiantes, se pueden distinguir cuatro enfoques, las investigaciones centradas en el cambio de la presentación del contenido, las investigaciones centradas en un cambio del modelo de evaluación, las investigaciones que usan modelos cognitivos para estudiar la comprensión de las demostraciones y estudios que abordan la comprensión de la demostración en la educación superior.

\section{Investigaciones centradas en el cambio de presentación de contenido}

Leron (1983) observó que las demostraciones matemáticas que se presentan en los libros de texto y artículos de revista son lineales, es decir, van desde una hipótesis hasta la conclusión, sin embargo, desde su experiencia cómo docente universitario, observó que los estudiantes no son capaces de descubrir cuál es la estructura que se encuentra implícita en una demostración, siendo esté un elemento importante para su comprensión. Por esto, propone un método alternativo, denominado método estructural con el cual pretende aumentar la comprensibilidad de las demostraciones matemáticas sin perder su rigor. Esta metodología consiste en organizar la información otorgada por demostración en tres niveles, de arriba hacia abajo; los niveles mismos consisten en módulos autónomos cortos. En el primer nivel se plasma la idea principal de la demostración, buscando dar un resumen o una visión global, por lo general suele consistir en la construcción de un nuevo objeto intermedio que está directamente conectado con todas sus partes, ofrece un punto de vista de ver la arquitectura global de la demostración. El principal beneficio de las presentaciones en el estilo estructural es que las ideas que hay detrás de las demostraciones se comuniquen mejor.

Con el tiempo, Rowland (2001) expone que por regla general, se espera que los estudiantes adquieran el conocimiento de los procedimientos de demostración matemática por un proceso de ósmosis, trayendo cómo consecuencia que la demostración sea inaccesible como dimensión de su vida matemática activa, concordando con Hersh (1993) el cual sostiene que, en el contexto de enseñanza, el propósito principal de la demostración es explicar, iluminar por qué algo es así, más que tener la seguridad de que es así. Por esto propone las demostraciones genéricas en el aprendizaje de la teoría de números, las cuales define cómo seleccionar cuidadosamente un ejemplo particular que permita ver la estructura general de la demostración, junto con el uso de ejemplos genéricos, que son una aplicación real de la demostración, pero presentados de tal manera que pone en manifiesto su función de portador de lo general como posible estrategia para la enseñanza de las demostraciones matemáticas.

Recientemente con la idea de entrelazar los recursos tecnológicos con las demostraciones matemáticas Alcock (2009) propone un medio interactivo denominado e-proof para presentar la demostración, las cuales consisten en una secuencia de pantallas donde: cada pantalla muestra el teorema y la demostración completa, pero gran parte de esto está atenuado para enfocar la atención en líneas particulares resaltando sus relaciones y enlaces lógicos entre ellas. Cada pantalla es también acompañada de un archivo de audio que replicará la explicación dada de forma expositiva por el docente en el aula. En estudios posteriores la autora junto con otros investigadores, encontraron que de manera inmediata no era muy significativa la comprensión lograda con respecto a estudiantes que solo leían las demostraciones de los libros; y a largo plazo, empeoran sus resultados, ya que encontraban muy clara e inmediata la información en la demostración, lo que reducía las horas de estudio por parte de los estudiantes. 


\section{Investigaciones centradas en el cambio del modelo de evaluación}

Conradie y Frith investigadores de University of Cape Town, en el año 2000 publicaron un artículo en donde señalan que observaron las formas tradicionales de evaluación generalmente conducen a estrategias de aprendizaje de memoria con poca comprensión. Además de destacar la importancia de la comprensión de las demostraciones, resaltan que su ventaja está en la mejora de la calidad de retroalimentación tanto para el estudiante como para el profesor de esta forma de evaluación.

Por su parte Yang y Lin (2007) plantearon un modelo que trata sobre la comprensión de las demostraciones en geometría al nivel de la educación en secundaria, este modelo está compuesto de cuatro niveles: En el primer nivel denominado superficie, los estudiantes adquieren conocimientos básicos sobre el significado de declaraciones y símbolos en la demostración. El segundo le llamaron reconociendo los elementos y los estudiantes identifican el estado lógico de los enunciados que fueron utilizados explícita o implícitamente en la demostración. El tercer nivel denominado encadenando los elementos, los estudiantes comprenden la forma en que estos diferentes enunciados están conectados en la demostración identificando las relaciones lógicas entre ellos; y el cuarto nivel al que se refirieron como encapsulación en el que median si los estudiantes interiorizan la demostración como un todo al reflexionar sobre cómo se puede aplicar la prueba a otros contextos. Sin embargo, Yang y Lin (2007) indicaron que su instrumento para medir a los estudiantes no tenía como objetivo diagnosticar si un estudiante había alcanzado este nivel superior.

Mejia Ramos et al. (2012) tomaron como base el trabajo realizado por Conradie y Frith (2000) y el trabajo de Yang y Lin (2007), para dar soporte a un modelo teórico de evaluación de la comprensión de las demostraciones a un nivel de educación superior. Los autores han proporcionado un modelo de evaluación para la comprensión de la demostración y, por lo tanto, describieron la comprensión de la demostración en términos pragmáticos incluyendo desde una comprensión local hasta una comprensión holística. Así, comprender una demostración desde la comprensión local implica que los estudiantes escriban el enunciado del teorema en sus propias palabras. Que conozcan las definiciones y los términos clave en ella. Que conozcan el estado lógico de los enunciados que están presentes. Así como conocer el tipo de marco de demostración (por ejemplo, directo, contrapositivo, contradicción, inducción). También implica saber el cómo o por qué cada declaración se deriva de declaraciones anteriores (por ejemplo, hacer explícitas garantías implícitas). Por otro lado, la comprensión holística incluye ser capaz de resumir sus ideas principales o claves. Identificar sub-demostraciones y cómo están relacionadas con la estructura general. Instanciar sus partes difíciles con un ejemplo para ayudar a la comprensión. Proporcionar un resumen usando las ideas de esa demostración en otra similar.

\section{Modelos cognitivos que trabajan la comprensión de las demostraciones}

En la búsqueda de literatura acerca de la comprensión de las demostraciones matemáticas se encuentran dos modelos teóricos que proponen formas de acercarse a la comprensión de las demostraciones a partir de modelos cognitivos.

El primero es la teoría APOS, cuya sigla traducida del inglés significa Acción, Proceso, Objeto y Esquema; fue creada por Ed Dubinsky (1996) está basada en teorías neopiagetianas que describen cognitivamente cómo los estudiantes construyen o aprenden conceptos en matemáticas con base en sus estructuras previas, que a su vez evolucionan para formar otras formas de conocimientos (Arnon, Cottrill, Dubinsky, Oktaç, Roa Fuentes, Trigueros y Weller, 2014). Así, una acción es una transformación de objetos percibidos por el individuo como esencialmente externos y como requiriendo, ya sea explícitamente o de 
memoria, instrucciones paso a paso sobre cómo realiza la operación. Cuando se repite una acción y el individuo reflexiona sobre ella, puede hacer una construcción mental llamada proceso en el que el individuo puede pensar que realiza el mismo tipo de acción, pero ya no con la necesidad de estímulos externos. Un objeto se construye a partir de un proceso cuando el individuo se da cuenta del proceso como una totalidad y cuando el individuo se da cuenta de él como un total y reconoce el efecto que las transformaciones podrían tener en sí mismo. Finalmente, un esquema para un cierto concepto matemático es la colección de acciones de un individuo, procesos, objetos y otros esquemas que están vinculados por algunos principios generales para formar un marco en la mente del individuo que puede aplicarse a una situación problemática que involucra ese concepto. Esta teoría proporciona, además, un ciclo de investigación compuesto por tres componentes: análisis teórico o descomposición genética, diseño y aplicación de instrumentos y análisis y verificación de los datos.

El segundo se refiere a los esquemas de demostración de Harel y Sowder (1998), quienes, basándose en el componente social de las demostraciones, el concepto de conjetura y lo que es determinar y persuadir para el individuo, crearon los esquemas de demostración. Estos consisten en la organización de los esquemas cognitivos que presentan los estudiantes al momento de construir una demostración. Cabe resaltar que se encuentran estructurados en tres categorías: convicción externa, demostración empírica y demostración analítica; el primero está relacionado con los hábitos que priorizan el seguimiento de fórmulas para resolver problemas y aprendiendo de memoria, sumado a que el docente sea visto cómo la única fuente de conocimiento; el siguiente está relacionado cuando los estudiantes comprueban por sí mismos y persuaden a otros sobre la veracidad de una conjetura, evaluando cuantitativamente la conjetura en uno o más casos concretos; por último, la demostración analítica consta de las conjeturas mediante deducciones lógicas, lo que en otras palabras es realizar demostraciones matemáticas. Para estos autores, cada una de las categorías de esta clasificación representa una etapa cognitiva en el desarrollo matemático de los alumnos.

\section{Estudios que abordan la comprensión de la demostración en la educación superior}

Anteriormente se había mencionado que aún no existe un consenso sobre lo que significa comprender una demostración, sumado a que MejiaRamos y Inglis (2009) en su artículo destacan que encontraron poca literatura en educación matemática relacionada con esta actividad; situación que hizo develar el interés en investigadores conllevando la realización de estudios relacionados con: recopilar información centrada en el fenómeno, proponer modelos de evaluación, indagar sobre las estrategias que utilizan matemáticos expertos y estudiantes de alto rendimiento, comparar resultados entre el uso de una estrategia específica y la enseñanza tradicional.

Por ejemplo, en el estudio cuantitativo realizado por Hodds, Alcok y Inglis (2014), tenian por objetivo validar si el entrenamiento de la autoexplicación sería un método eficaz para mejorar la comprensión de las demostraciones de los estudiantes de pregrado, para ello realizaron tres experimentos, los cuales partían de impartir la capacitación de un entrenamiento de autoexplicación para luego validar su comprensión mediante el modelo de evaluación propuesto por Mejia-Ramos et al (2012). En los experimentos 1 y 3, realizaron sesiones similares, en el cual los estudiantes eran seleccionados aleatoriamente para hacer parte del grupo control y el grupo que inicialmente recibió el entrenamiento de autoexplicación mediante una serie de diapositivas que lo describen, luego ambos grupos leían la demostración y por último presentaban la prueba.

Cabe aclarar que en el experimento 1 evaluaron en la calidad de las explicaciones basándose en la 
categorización realizada por Ainsworth y Burcham (2007) mediante las grabaciones del pensamiento en voz alta, encontrando que el hecho de recibir capacitación en autoexplicación aumentó tanto el número como la proporción de explicaciones de alta calidad, dadas por los participantes durante sus intentos de comprensión de las demostraciones. A diferencia del experimento 3 que lo llevaron a cabo en un entorno verdaderamente pedagógico, donde sumado a lo anterior, realizaron una prueba retrasada veinte días después, encontrando que el entrenamiento de autoexplicación en un entorno pedagógico típico mejoró significativamente la comprensión de la demostración a corto plazo y tuvo efectos duraderos.

Desde otra perspectivarelacionadacon el estudio de la comprensión de las demostraciones, Weber (2015) realizó un estudio exploratorio en donde se propuso desarrollar hipótesis fundamentadas sobre qué estrategias de lecturas pueden ser útiles para esta actividad matemática. Como marco teórico utilizó el modelo de evaluación de la comprensión de las demostraciones propuesto por Mejia-Ramos et al (2012) para validar sus estrategias. En el estudio empleó una metodología cualitativa, seleccionando cuatro estudiantes de último año del programa de Licenciatura en Matemáticas con las condiciones de tener un alto rendimiento académico y que ya hayan participado en estudios anteriores realizados por el autor.

Para identificar tales estrategias, el autor realizó un registro en un entorno en el que probablemente se provoquen las estrategias. Por esta razón, eligió grabar en video articulaciones exitosas en matemáticas mientras pensaban en voz alta al tiempo que leían seis demostraciones de cálculo y teoría de números básica, con las condiciones de que cuya veracidad no sería inmediatamente obvia para un estudiante universitario y que emplea una técnica de demostración interesante con la que esperaba que los participantes no hubieran tenido una amplia experiencia. Es importante resaltar que en cada prueba se basó en el modelo de comprensión de Mejía-Ramos et al (2012) para generar el conjunto de preguntas. Como hay pocos estudios empíricos sobre comprensión de demostración (Mejía-Ramos y Inglis, 2009), optó por utilizar un esquema de codificación abierto al estilo de Strauss y Corbin (1990).

Esta investigación arrojó como resultado que se hallaron seis estrategias de lectura, estas son: estrategia no 1. Comprender el enunciado del problema; la estrategia no. 2. Intente probar el enunciado del teorema antes de leer su demostración; estrategia no 3. Considerar el marco de demostración utilizado; estrategia no 4. Dividir la demostración en partes o sub-demostraciones; estrategia no 5. Usar ejemplos para dar sentido a las declaraciones dentro de la demostración; estrategia no 6. Comparar el método de la demostración con los métodos propios.

Partiendo de los hallazgos de los anteriores estudios Neuhaus y Rach (2019) realizaron una investigación donde tenían por objetivos encontrar la correlación entre las las estrategias de lectura encontradas por Weber (2015), el entrenamiento de autoexplicación de Hodds et al. (2014) y el modelo de evaluación de Mejia- Ramos et al (2012) y por otra parte, también se centraron en la correlación de sus características individuales (como el autoconcepto, el interés o el conocimiento previo) y la comprensión de las demostraciones . Para realizar la investigación tomaron cómo muestra 64 estudiantes en segundo semestre de una universidad en Alemania, donde todos los estudiantes habían asistido a las mismas conferencias, donde el contenido incluye análisis matemático.

En la investigación utilizaron dos instrumentos para revisar el uso de las estrategias de lectura, el primer instrumento es una prueba de comprensión que constaba de 10 preguntas sobre el teorema del valor medio, donde inicialmente fue revisado por expertos y posterior a ello se tomaron en cuenta 
las correcciones para mejorar la prueba; el segundo instrumento fue una escala Likert con 22 ítems que cubren diferentes tipos de estrategias de lectura, usando ideas de una prueba de comprensión lectora en alemán, el entrenamiento de autoexplicación de Hodds et al. (2014), y las estrategias de lectura efectivas esperadas (1 - 3) del estudio de Weber (2015). Además, para medir las características individuales los estudiantes se sometieron a una prueba para examinar sus conocimientos previos en análisis (Rach y Heinze, 2017), sumado a las preguntas sobre sus calificaciones en matemáticas al finalizar su etapa escolar y curso de análisis matemático, interés en las matemáticas escolares y universitaria, su autoconcepto en relación con la prueba, a la escuela respectivamente la matemática universitaria en general y su satisfacción referente a su carrera universitaria.

En el análisis de los resultados no encontraron correlaciones positivas entre la comprensión de las demostraciones y el interés de los estudiantes por las matemáticas universitarias o su autoconfianza matemática, sugiriendo que el interés en las matemáticas escolares y el interés en las matemáticas universitarias deben analizarse por separado. En las pruebas relacionadas con las estrategias encontraron que utilizaban una de las estrategias de Weber (2015) y ninguna basada en el entrenamiento de autoexplicación de Hodds et al (2014), esto les hace concluir que las estrategias deben ser entregadas y practicadas por los estudiantes con anticipación para que puedan beneficiarse más de las pruebas.

Por otra parte, Kolahdouz, Radmehr, y Alamolhodaei, (2019) observaron que los estudiantes universitarios iraníes presentaban dificultades que estaban en concordancia con los estudios anteriormente mencionados, relacionadas con el aprendizaje memorístico de los teoremas y las demostraciones matemáticas, por esto realizaron una exploración de la comprensión relacionados con la demostración del Teorema del Valor Medio Generalizado de Cauchy. Para llevar a cabo la investigación se basaron en el modelo de evaluación diseñado por Mejia- Ramos et al, para diseñar su prueba.

El estudio utilizó una metodología mixta, en el enfoque cuantitativo participaron 35 estudiantes que cursan Cálculo de una especialización de matemáticas, suministrándoles una prueba de comprensión y luego a 10 de ellos les realizaron una entrevista; luego realizaron un análisis inductivo, utilizando una codificación abierta, axial y selectiva. Cabe resaltar que en los resultados encontraron que los estudiantes no estaban preparados para responder ese tipo de preguntas, ya que tendieron a memorizar las pruebas y enfrentaban dificultades al responder las preguntas que estaban fuera del aprendizaje memorístico.

Algunos estudios más recientes han abarcado este tema buscando la relación de la comprensión de las demostraciones con el razonamiento cuantitativo. Así, Belin y Akar (2020), investigadores de Boğaziçi University, partían de las dificultades que tanto estudiantes como profesores tienen en los procesos de demostración. Tales dificultades incluyen la falta de conocimiento sobre las definiciones de términos y declaraciones, y cómo usarlos en la demostración; falta de comprensión de los conceptos y la falta de generar y usar los propios ejemplos de los estudiantes sobre las declaraciones de la demostración (Moore, 1994). Por ello su objetivo de investigación fue investigar el efecto del razonamiento cuantitativo en la comprensión de los futuros profesores de matemáticas de una demostración en números reales. Su soporte teórico está en la teoría del razonamiento cuantitativo (Thompson, 1994) y en el modelo de evaluación de Mejia-Ramos et al. (2012).

Para realizar este trabajo, usaron un enfoque mixto con un diseño experimental integrado en el que tomaron a 19 participantes a los que le aplicaron pruebas de comprensión antes y después de una instrucción sobre métodos de enseñanza, la instrucción fue de cinco horas separada en dos sesiones, en la que trabajaron el tema de los 
números reales. Posteriormente a seis participantes les aplicaron entrevistas semiestructuradas. Para cuestiones de validez sometieron las pruebas de comprensión a ser validadas por dos matemáticos y un educador de matemáticas. Como conclusión los investigadores encontraron progreso en las habilidades de comprensión desde la prueba previa a la posterior.

\section{Conclusiones}

En la búsqueda de artículos referidos al núcleo temático de la investigación, es importante resaltar que existe una cantidad limitada en idioma español en comparación con los artículos en inglés. A pesar de que se utilizaron descriptores en español en las bases de datos, los resultados generalmente se relacionaban con la utilización de estrategias didácticas que se enfocan en la construcción o validación de las demostraciones.

También se encontró importante hacer una distinción al concepto de comprensión de la demostración, pues la mayoría de trabajos previos a los modelos de evaluación asumen la comprensión a partir de evaluar la forma en cómo los estudiantes construían demostraciones o validaron estas, pero desde un enfoque memorístico prestando poca atención a la comprensión lectora que poseen los estudiantes.

Al interior de los programas de pregrado que incluyen asignaturas cuyo objeto de estudio son las demostraciones, es menester que se generen estrategias didácticas y fomente el uso de modelos de evaluación que promuevan la comprensión. En concordancia con Hersh (1993), su uso debe tener cómo propósito comunicar y exponer cómo y porqué las generalizaciones y reglas utilizadas funcionan para hacer representaciones de la realidad, con la finalidad de que los estudiantes puedan resolver problemas; pero, esto solo es posible si ellos alcanzan a comprender cómo se construyen y cómo se relacionan con otras demostraciones. Por lo anterior es importante que en el desarrollo de los estudios de pregrado dicho contenido sea llevado a cabo desde un enfoque que promueva la comprensión.

De lo anterior, se propone la creación de líneas de investigación que tengan como objeto de estudio la comprensión de las demostraciones, desde el enfoque de los modelos de evaluación que se centran en lograr en los estudiantes una comprensión local y holística de una demostración dada, pues se considera que para programas de pregrado deberían tener similar importancia que las demás actividades demostrativas.

\section{Referencias}

Ainsworth, S., \& Burcham, S. (2007). The impact of text coherence on learning by self-explanation. Learning and Instruction, 17(3), 286-303. doi: 10.1016/j.learninstruc.2007.02.004

Alcock, L. (2009). e-Proofs: Student experience of online resources to aid understanding of mathematical proofs. In Proceedings of the 12th Conference on Research in Undergraduate Mathematics Education. Raleigh, NC: Special Interest Group of the Mathematical Association of America on Research in Undergraduate Mathematics Education

Arnon, I., Cottrill, J., Dubinsky, E., Oktaç, A., Roa Fuentes, S., Trigueros, M., \& Weller, K. (2014). APOS Theory. doi:10.1007/978-1-4614-7966-6

Balacheff, N. (1987). Processus de preuve et situations de validation (Proving Processes and Situations for Validation). Educational Studies in Mathematics, 18(2), 147-176. Retrieved January 18, 2021, from http://www.jstor.org/ stable/3482413

Belin, M., \& Akar, G. K. (2020). The effect of quantitative reasoning on prospective mathematics teachers' proof comprehension: The case of real numbers. The Journal of Mathematical Behavior, 57, 100757. doi: 
10.1016/j.jmathb.2020.100757

Ben-Zvi, D. \& Sfard, A. (2007). Ariadne's thread, Daedalus' wings, and the learner's autonomy. Education \& Didactique, 1(3), 117-134. Recuperado de: https://journals.openedition.org/ educationdidactique/241?lang=es\#

Conradie, J., \& Frith, J. (2000). Comprehension tests in mathematics. Educational Studies in Mathematics, 42(3), 225-235. Doi: https://doi. org/10.1023/A:1017502919000

Guirao-Goris,J.A; Olmedo Salas,A; Ferrer Ferrandis, E.(2008) El artículo de revisión. Revista Iberoamericana de Enfermería Comunitaria, 1, 1, 6. Disponible en http://revista. enfermeriacomunitaria.org/articuloCompleto. php?ID=7. Consultado el 23/07/2008

Harel, G. \& Sowder, L. (1998). Students' proof schemes: Results from exploratory studies. American Mathematical Society, 7, 234-283. Recuperado de: https://math.ucsd.edu/ harel/ Students $\% 27 \% 20$ Proof $\% 20$ Schemes.pdf

Harel, G. \& Sowder, L. (2007) Toward Comprehensive Perspectives on the Learning and Teaching of Proof. In: Lester, F., Ed., Second Handbook of Research on Mathematics Education. Recuperado de: https://math.ucsd. edu/ harel/TowardComprehensivePerspective. pdf

Hersh, R. (1993). Proving is convincing and explaining. Educational Studies in Mathematics, 24(4), 389-399

Hodds, Alcock, \& Inglis. (2014). Self-Explanation Training Improves ProofComprehension. Journal for Research in Mathematics Education, 45(1), 62-101. doi:10.5951/jresematheduc.45.1.0062

Kolahdouz, F., Radmehr, F., \& Alamolhodaei, H.
(2019). Exploring students' proof comprehension of the Cauchy Generalized Mean Value Theorem. Teaching Mathematics and Its Applications: An International Journal of the IMA, 1-20. doi:10.1093/teamat/hrz016

Leron, U. (1983). Structuring mathematical proofs. American Mathematical Monthly, 90(3), 174184

Mejia-Ramos, J. P., Fuller, E., Weber, K., Rhoads, K., \& Samkoff, A. (2012). An assessment model for proof comprehension in undergraduate mathematics. Educational Studies in Mathematics, 79(1), 3-18. doi:10.1007/s10649011-9349-7

Mejia-Ramos, J. P., \& Inglis, M. (2009). Argumentative and proving activities in mathematics education research. In F.-L. Lin, F.-J. Hsieh, G. Hanna, \& M. Recuperado de: http://140.122.140.1/ icmi19/files/Volume_2. pdf

Moore, R. C. (1994). Making the transition to formal proof. Educational Studies in Mathematics, 27(3), 249-266. doi:10.1007/bf01273731

Neuhaus, S., \& Rach, S. (2019). Proof comprehension of undergraduate students and the relation to individual characteristics. In Eleventh Congress of the European Society for Research in Mathematics Education (No. 31). Freudenthal Group; Freudenthal Institute; ERME

Pfeiffer, K. (2011). Features and purposes of mathematical proofs in the view of novice students: observations from proof validation and evaluation performances (Doctoral dissertation, National University of Ireland, Galway)

Rach, S., \& Heinze, A. (2017). The transition from school to university in mathematics: Which influence do school-related variables 
have. International Journal of Science and Mathematics Education, 15(7), 1343-1363. https://doi.org/10.1007/s10763-016-9744-8

Real Academia Española. (s.f.). Comprensión. En Diccionario de la lengua española Recuperado en 01 de abril 2021, de https://dle.rae.es/ comprensi $\% \mathrm{C} 3 \% \mathrm{~B} 3 \mathrm{n}$

Rowland, T. (2001). Generic proofs in number theory. In S. Campbell and R. Zazkis (Eds.), Learning and teaching number theory: Research in cognition and instruction. (pp. 157-184). Westport, CT: Ablex Publishing

Sánchez Upegui, A. A. (2011). Manual de redacción académica e investigativa: cómo escribir, evaluar y publicar artículos. Fundación Universitaria Católica de Norte

Selden, A., \& Selden, J. (2017). A comparison of proof comprehension, proof construction, proof validation and proof evaluation. In Proceedings of the Conference on Didactics of Mathematics in Higher Education as a Scientific Discipline (pp. 339-345)

Schoenfeld, A. H. (1988). When good teaching leads to bad results: The disasters of well-taught mathematics courses. Educational psychologist, 23(2), 145-166

Sparks, J. R. (2012). Language/Discourse Comprehension and Understanding. In Encyclopedia of the Sciences of Learning (pp. 1713-1717). Norbert M. Seel. https://doi. org/10.1007/978-1-4419-1428-6_100

Strauss, A., \& Corbin, J. M. (1990). Basics of qualitative research: Grounded theory procedures and techniques. Sage Publications, Inc

Thompson, P. W. (1994). The development of the concept of speed and its relationship to concepts of rate. In G. Vergnaud, G. Harel, \& J. Coufrey (Eds.). The development of multiplicative reasoning in the learning of mathematics (pp. 179-234). SUNY Press.

Weber, K., \& Mejia-Ramos, J. P. (2011). Why and how mathematicians read proofs: an exploratory study. Educational Studies in Mathematics, 76(3), 329-344. doi:10.1007/s10649-0109292-z

Weber, K. (2015). Effective Proof-Reading Strategies for Comprehending Mathematical Proofs. International Journal of Research in Undergraduate Mathematics Education, 1(3), 289-314. doi:10.1007/s40753-015-0011-0

Yang, K.-L., \& Lin, F.-L. (2007). A model of reading comprehension of geometry proof. Educational Studies in Mathematics, 67(1), 5976. doi:10.1007/s10649-007-9080-6 